\title{
RELACIÓN DE LOS ESTILOS INTERPERSONALES DEL PROFESOR Y LA MOTIVACIÓN, DIVERSIÓN Y ABURRIMIENTO DEL ESTUDIANTE EN LA CLASE DE EDUCACIÓN FÍSICA EN ESCUELAS SECUNDARIAS DE HERMOSILLO, SONORA, MÉXICO
}

\author{
RELATIONSHIP OF THE INTERPERSONAL STYLES OF THE TEACHER \\ AND THE MOTIVATION, FUN AND BOREDNESS OF THE STUDENT IN \\ THE PHYSICAL EDUCATION CLASS IN HERMOSILLO HIGH SCHOOLS, \\ SONORA, MEXICO
}

\begin{abstract}
Duarte Félix Héctor ${ }^{1}$, Castro Zamora Andrés Aquilino² ${ }^{2}$ Borbón Castro Norma Angélica ${ }^{3}$, De La Cruz-Ortega Manuel Francisco ${ }^{4}$, Durazo Terán Luis Alberto ${ }^{5}$.
\end{abstract}

\begin{abstract}
${ }^{1} \mathrm{https}: / /$ orcid.org/0000-0002-1960-1995 Universidad Estatal de Sonora. 2https://orcid.org/0000-0002-4938-3597 Universidad Estatal de Sonora. 4 https://orcid.org/0000-0002-6687-7775 Universidad Estatal de Sonora 5https://orcid.org/0000-0003-0473-0126 Universidad Estatal de Sonora
\end{abstract}

\section{https://doi.org/10.46589/rdiasf.vi36.410}

Recibido 7 de julio 2021.

Aceptado 30 de septiembre 2021

Publicado 1 de noviembre 2021

\section{Resumen}

El objetivo de este estudio fue analizar el papel predictor del estilo interpersonal de los profesores de educación física sobre los tipos de motivación y éstos a su vez con el aburrimiento y diversión en estudiantes de secundarias de Hermosillo, Sonora. Participaron un total de 282 estudiantes $(55.3 \%$ chicos, $44.7 \%$ chicas, Medad $=12.64$; DT $=.66$; rango $=12-14$ años). Los resultados mostraron una relación positiva entre el apoyo a la autonomía y motivación autónoma, así como una relación positiva con la satisfacción / diversión y negativamente la motivación controlada y la no motivación, así como con el aburrimiento, mientras que el estilo controlador tuvo una relación negativa con la motivación autónoma y la satisfacción / diversión y una relación positiva con la motivación controlada y la no motivación además del aburrimiento. Asimismo, este estudio tiene algunas limitaciones, ya que solo se pudo aplicar en una escuela, por lo que sería interesante ampliar el estudio y aplicarlo en más escuelas secundarias. 
Palabras clave: Educación física, Motivación, Aburrimiento, Diversión, Estilo Interpersonal.

\begin{abstract}
The objective of this study was to analyze the predictive role of the interpersonal style of physical education teachers on the types of motivation and these in turn with boredom and fun in high school students from Hermosillo, Sonora. A total of 282 students participated (55.3\% boys, $44.7 \%$ girls, Average $=12.64 ; \mathrm{SD}=.66$; range $=12-14$ years). The results showed a positive relationship between support for autonomy and autonomous motivation, as well as a positive relationship with satisfaction / fun and negatively controlled motivation and nonmotivation, as well as with boredom, while the controlling style had a relationship negative with autonomous motivation and satisfaction / fun and a positive relationship with controlled motivation and non-motivation in addition to boredom. Likewise, this study has some limitations, since it could only be applied in one school, so it would be interesting to expand the study and apply it in more secondary schools.
\end{abstract}

KEY WORDS: Physical education, Motivation, Boredom, Fun, Interpersonal style.

\title{
Introducción
}

La clase de Educación Física (EF) resulta ser el medio ideal para que el profesorado pueda fomentar hábitos de vida saludables entre los estudiantes de EF, ya que esta asignatura tiene su foco de atención en la implementación de propuestas académicas para el desarrollo de competencias que tiene como finalidad la promoción de hábitos de vida saludables en virtud de la práctica regular de actividad física (Vázquez y Márquez, 2017; Zueck et al., 2020). Para ello, se requiere de un papel activo por parte del docente, cuya interacción con el alumnado se torna indispensable para que esté consiga entender los beneficios de la actividad física a nivel psicológico, conductual y social, consolidando hábitos activos y alimenticios saludables (Márquez y Celis, 2016; Tapia, 2019).

La Teoría de la Autodeterminación (TAD; Deci y Ryan, 2008) sugiere que la actitud que pueda adoptar el docente durante la clase de EF refiriéndose al alumnado se puede analizar desde varias perspectivas,como el apoyo a la autonomía o conductas que buscan el control absoluto del alumnado. El primero hace referencia a que el alumno tome la decisión por si solo 
para su aprendizaje y la mejora de sus propias habilidades (Cheon et al., 2014; Janget al., 2010; Rius y Torrebadella, 2018). Por el contrario, las conductas controladoras del docente sobre el alumno muchas veces los abruman porque sienten que el profesor solo quiere que se haga lo que él dice en la clase EF y se sienten controlados (Bartholomew et al., 2011).

El apoyo a la autonomía es la entrega de instrucción a través de un tono interpersonal de apoyo y comprensión que aprecia, vitaliza y respalda las necesidades psicológicas de los estudiantes en cuanto a autonomía, competencia y relación (Reeve, 2016). Este tono se comunica a los estudiantes a través de una prosodia de apoyo es decir, tono más alto, velocidad de habla lenta, calidad de voz leve; (Zougkouet al., 2017) y actos de instrucción como tomar la perspectiva de los estudiantes, crear oportunidades. Así mismo, el apoyo de autonomía proporcionado por el profesor es también citado con la motivación de alta calidad de los estudiantes (necesidad de satisfacción, motivación autónoma), funcionamiento efectivo del aula (compromiso, aprendizaje conceptual) y resultados educativos positivos (alto rendimiento, bienestar) y, por lo tanto, se considera el aspecto adaptativo del estilo motivador de un maestro (Assor et al., 2002; Cheon y Reeve, 2015; Gunnell et al., 2013; Jang et al., 2016).

\section{Materiales y Método}

Se presenta un estudio de tipo no experimental, con un enfoque cuantitativo, realizando un estudio descriptivo-correlacional, transversal y de campo, para identificar y analizar el papel predictor del apoyo a la autonomía por parte del profesor de EF, como predictor de la motivación y de la satisfacción/diversión en estudiantes de escuelas secundarias de Hermosillo, Sonora.

\section{- Teoría de la autodeterminación.}

Cuando se habla de macro-teoría explicativa basada en el comportamiento de las personas y el desarrollo de personalidad; se hace referencia a la teoría de la autodeterminación. En está el análisis de la TAD está centrado principalmente a nivel psicológico, diferenciado así los tipos de motivación a lo largo de un continuo controlador a un autónomo (Decy y Ryan, 1985). Otro de los aspectos importantes en los que está basada esta hipótesis, es en como los factores socio- contextuales apoyan a que las personas prosperen a través de la satisfacción de sus necesidades centrándose así en las psicológicas básicas de competencia, relación y autonomía. Sin embargo, aunque este centrada en el ámbito psicológico, retoma y pone atención en los fundamentos biológicos de estos mismos. 


\section{- Apoyo a la autonomía.}

En el medio escolar, el apoyo a la autonomía ocupa un lugar muy importante, ya que se refiere al esfuerzo de instrucción para proporcionar a los alumnos un ambiente de aula y una relación profesor-alumno que preserve la necesidad de autonomía de sus educandos. Lo que hace que se desarrollen, fortalezcan y crezcan los recursos de motivaciones internos de los estudiantes son, el apoyo a la autonomía que se refleja como un sentimiento y conducta interpersonal que el docente proporciona durante la instrucción, para identificar, vitalizar y nutrir los mismos.

Reeve (2009) plantea cinco comportamientos de instrucción que los profesores pueden adoptar para ser más autosuficientes: (a) fomentar la motivación intrínseca, (b) ofrecer justificación para las tareas, (c) utilizar lenguaje informativo / no controlador, (d) mostrar paciencia para el aprendizaje del estudiante y (e) reconocer y aceptar las expresiones de los estudiantes de afecto negativo.

\section{- Estilo Controlador.}

Un estilo de control puede expresarse en al menos dos maneras diferentes según la TAD, es decir, como una enseñanza externa o interna (Ryan, 1982, Soenens y Vansteenkiste, 2010; Vansteenkiste, et al., 2005). Los docentes caóticos crean desconcierto entre los estudiantes al ejercer una estructura ilógica e incoherente al realizar tareas y expresar comentarios ambiguos o incluso críticas destructivas (Reeve y Jang, 2006).

Cuando el profesor maneja un estilo controlador, esté hace uso de un sentimiento interpersonal y conductual que proporciona en la instrucción para presionar a los estudiantes para que piensen, sientan o se comporten de la manera que el docente desea (Reeve, 2009). El guiar a los estudiantes es una herramienta muy poderosa, en la práctica los maestros muchas veces frustran a sus discípulos con formas de controlar, las cuales terminan alejándolos de lo que les gusta, el objetivo es que el educando logre sentir, pensar y pueda opinar durante la clase de educación física.

\section{- La motivación.}

En la temática de la motivación podemos encontrar a la TAD, la cual está basada en que todos los seres humanos, tienden a crecer e integrar desde las experiencias de vida en curso 
(Deci y Ryan, 2000); Como se ha planteado, según la TAD, existen tres tipos distintos de motivación (no motivación o amotivación, motivación extrínseca y motivación intrínseca) estas conducen a diferentes resultados afectivos, cognitivos y de comportamiento basados en el grado en que el comportamiento ha sido Interiorizado e integrado en el yo.

La orientación de la motivación más auto determinada es la regulación intrínseca, donde el placer derivado de la conducta se encuentra en el comportamiento mismo. Se caracteriza por un compromiso espontáneo en la actividad alimentada por el interés, la curiosidad y la dificultad de la tarea (Deci y Ryan, 2002).

\section{- La no motivación (amotivación).}

Cuando no existe una intención de actuar, se observan sujetos que no son capaces de percibir una contingencia entre sus conductas y los resultados subsecuentes de su conducta (Deci y Ryan, 1985b), debido a que, no hay intención ni sentido de causa (Ryan y Deci, 2000).

Esta se presenta cuando el individuo no tiene ninguna intención de realizar la actividad, ya sea, porque no se siente capaz para ello o al no creer que conseguirá el resultado deseado. Una de las causas puede ser que la actividad programada esté desorganizada, o que este asociado con sentimientos de frustración, miedo o depresión. Cuando una persona está no motivada, simplemente no actúa o actúa pasivamente, es decir, ellos realizan los movimientos sin intención de hacer lo que están haciendo (Deci y Ryan, 2002).

\section{- Estilos interpersonales del profesor.}

Para medir el apoyo a la autonomía por parte del profesor se utilizó la versión corta del LCQ de Williams y Deci (1996) adaptada y validada al contexto mexicano por Maldonado et al., (2017) se puede visualizar en tabla 3. Por su parte, el estilo controlador del profesor se midió con los cuatro ítems de la Escala de Profesor Controlador (Teacher Controllingness Scale [TCS]) de Jang et al., (2010) (Tabla 1). En las instrucciones se pide que indiquen el grado de acuerdo con los ítems, recogiéndose las respuestas en una escala de ítems politómicos de 7 puntos que oscila desde Muy en desacuerdo (1) a Muy de acuerdo (7).

La adaptación al castellano del TCS se realizó atendiendo a los estándares metodológicos internacionales recomendados por la International Test Comision para adaptar correctamente test y escalas de unas culturas a otras (Muñiz, 2000; Muñiz y Bartram, 2007). Para evitar imprecisiones, se combinaron los diseños de traducción directa e inversa de los 
ítems, según el procedimiento parallel back translation (Brislin, 1986). La evaluación cualitativa de ítems (validez de contenido) se efectuó mediante el juicio de cuatro expertos (Osterlind, 1989): dos en construcción de escalas y dos conocedores del constructo a evaluar. Todos los ítems fueron analizados y revisados hasta que se recogiese la dimensión teórica de la forma más clara y precisa. El encabezado fue: “En esta clase de educación física...”. La nueva versión fue administrada a una muestra de 50 estudiantes de secundaria con edades entre 12 y 18 años. Los comentarios sobre instrucciones y forma de redacción supusieron cambios menores y tras una última revisión por el equipo de investigación, se llegó a la versión final del TCS adaptado a la EF (TCS-EF).

\section{Tabla 1}

Ítems que componen el LCQ y TCS

En mi clase de Educación Física...

Apoyo a la autonomía

1. Siento que mi profesor me brinda opciones y alternativas

2. Me siento comprendido por mi profesor

3. Mi profesor me transmite confianza sobre mi capacidad para realizar bien las actividades durante el curso

4. Mi profesor me motiva a que haga las preguntas

5. Mi profesor escucha como me gustaría hacer las cosas

6. Mi profesor trata de comprender como veo las cosas antes de sugerir una nueva forma de hacerlas

Estilo controlador

7. Mi profesor trata de controlar todo lo que hago

8. Mi profesor es inflexible

9. Mi profesor utiliza un lenguaje fuerte

10. Mi profesor pone demasiada presión sobre mi

- Motivación.

Los diferentes tipos de motivación de los estudiantes se midió con la Escala de Percepción de Locus de Causalidad (Perceived Locus of Causality Scale) de Goudas, et al., (1994) adaptada y validada al contexto mexicano por Zamarripa, et al., (2016).

La escala está compuesta de 20 ítems (Tabla 2) precedidos por el siguiente encabezado "Participo en las clases de educación física..." y compuesta por un total de 20 ítems (cuatro por 
factor), que miden la motivación intrínseca (e.g., "porque la educación física es divertida"), regulación identificada (e.g., "porque quiero aprender habilidades deportivas”), regulación introyectada (e.g., "porque me sentiré mal conmigo mismo si no lo hago"), regulación externa (e.g., "porque es lo que se supone que debo hacer") y no motivación (e.g., "pero en realidad siento que pierdo mi tiempo en esta clase"). El instrumento se responde sobre una escala Likert de 1 (totalmente en desacuerdo) a 7 (totalmente de acuerdo).

Tabla 2

Ítems que componen el PLOC

Participo en la clase de Educación Física...

Motivación Intrínseca

1. Porque es divertida

2. Porque me gusta aprender nuevas habilidades

3. Porque es emocionante

4. Porque disfruto como me siento mientras aprendo nuevas habilidades/técnicas

Regulación Identificada

5. Porque quiero aprender habilidades deportivas

6. Porque es importante para mi hacerlo bien

7. Porque quiero mejorar en los deportes

8. Porque puedo aprender habilidades que tal vez puedan ayudarme en otras áreas de mi vida

Regulación Introyectada

9. Porque quiero que el maestro piense que soy buen estudiante

10. Porque me sentiré mal conmigo mismo si no lo hago

11. Porque quiero que mis compañeros piensen que soy hábil

12. Porque me incomoda cuando no lo hago

Regulación Externa

13. Porque me meteré en problemas si no lo hago

14. Porque es lo que se supone debo hacer

15. Para que el (la) maestro (a) no me grite

16. Porque es parte del reglamento

No motivación

17. Pero realmente no sé por qué lo hago

18. Pero no entiendo por qué debemos tener esta clase

19. Pero en realidad siento que pierdo mí tiempo en esta clase

20. Pero no veo lo que estoy obteniendo de esta clase 


\section{- Satisfacción intrínseca}

Para medir satisfacción/diversión y aburrimiento se utilizó la Escala de Satisfacción Intrínseca en el Deporte de Balaguer, et al., (1997) adaptada al contexto de la educación física por Baena-Extremera, et al., (2012). El SSI-EF consta de 8 ítems (Tabla 3) para medir la satisfacción intrínseca mediante dos subescalas que miden satisfacción/diversión (5 ítems) y aburrimiento (3 ítems) en la clase de educación física. En las instrucciones se pide a los sujetos que indiquen su grado de acuerdo con los ítems que reflejan criterios de diversión o aburrimiento, recogiéndose las respuestas en una escala de ítems politómicos de 5 puntos que oscila desde muy en desacuerdo (1) a muy de acuerdo (5).

Tabla 3

Ítems que componen el SSI

\section{Satisfacción/diversión}

01 Normalmente me divierto en la clase de educación física

05 Normalmente encuentro la educación física interesante

06 Cuando hago educación física parece que el tiempo vuela

07 Normalmente participo activamente en las clases de educación física

08 Normalmente me lo paso bien haciendo educación física

Aburrimiento

02 En las clases de educación física a menudo sueño despierto en vez de pensar en lo que hago realmente

03 En las clases de educación física normalmente me aburro

04 En educación física deseo que la clase termine rápidamente 


\section{Resultados}

\section{Estilos interpersonales. Estadísticos descriptivos}

Tabla 4

Escala de estilos interpersonales. Descriptivos y normalidad

\begin{tabular}{|c|c|c|c|c|c|}
\hline No & Sub escala & M & DT & Asimetría & Curtosis \\
\hline \multicolumn{6}{|c|}{ Apoyo a la autonomía } \\
\hline 1 & $\begin{array}{l}\text {... siento que mi profesor me brinda opciones y } \\
\text { alternativas }\end{array}$ & 4.09 & 2.05 & -.03 & -1.14 \\
\hline 2 & ...me siento comprendido por mi profesor & 3.77 & 2.08 & .22 & -1.77 \\
\hline 3 & $\begin{array}{l}\text {...mi profesor me transmite confianza sobre mi } \\
\text { capacidad para realizar bien las actividades durante } \\
\text { el curso }\end{array}$ & 4.22 & 2.12 & -.12 & -1.27 \\
\hline 4 & ...mi profesor me motiva a que haga las preguntas & 3.09 & 2.09 & .56 & -.97 \\
\hline 5 & $\begin{array}{l}\text {...mi profesor escucha como me gustaría hacer las } \\
\text { cosas }\end{array}$ & 3.36 & 2.13 & .38 & -1.17 \\
\hline 6 & $\begin{array}{l}\text {...mi profesor trata de comprender como veo las } \\
\text { cosas antes de sugerir una nueva forma de hacerlas }\end{array}$ & 3.52 & 2.15 & .32 & -1.22 \\
\hline \multicolumn{6}{|c|}{ Estilo controlador } \\
\hline 7 & ...mi profesor trata de controlar todo lo que hago & 3.59 & 2.19 & .29 & -1.25 \\
\hline 8 & ...mi profesor es inflexible & 3.70 & 2.04 & .12 & -1.11 \\
\hline 9 & ...mi profesor utiliza un lenguaje fuerte & 2.74 & 2.05 & .83 & -.66 \\
\hline 10 & ...mi profesor pone demasiada presión sobre mi & 2.27 & 1.86 & 1.37 & -.68 \\
\hline
\end{tabular}

Nota. $\mathrm{M}=$ Media, DT = Desviación Típica. Rango: $1-7$.

En la Tabla 4 se muestran los resultados descriptivos de la escala de apoyo a la autonomía y estilo controlador. Los resultados de la asimetría y curtosis revelan una distribución no normal ya que la mayoría de los valores de asimetría y curtosis se encuentran fuera del rango $(-1,1)$. 


\section{- Análisis Factorial Exploratorio (AFE).}

Siguiendo las recomendaciones de estudios recientes (Lloret-Segura, et al., 2014), para examinar la estructura factorial de los 10 ítems que componen el instrumento para medir los estilos interpersonales se realizó un análisis factorial exploratorio utilizando el método de factorización de mínimos cuadrados no ponderados (unweighted least squares) porque funciona bien cuando se trabaja con muestras pequeñas, incluso, cuando el número de variables es elevado, especialmente si el número de factores a retener es pequeño (Jung, 2013) y aplicando un criterio de rotación Oblicua (concretamente Promax, Kappa $=4$ ) ya que teóricamente los dos factores se encuentran relacionados.

\section{Tabla 5}

Análisis factorial exploratorio de la escala de estilos interpersonales. Cargas factoriales

En la clase de educación física ...

Cargas

factoriales

\begin{tabular}{llcl} 
No & Ítem & AA & EC \\
\hline 1 & $\ldots$...siento que mi profesor me brinda opciones y alternativas & .78
\end{tabular}

2 ...me siento comprendido por mi profesor

...mi profesor me transmite confianza sobre mi capacidad para realizar

3 bien las actividades durante el curso

...mi profesor me motiva a que haga las preguntas

$5 \quad$...mi profesor escucha como me gustaría hacer las cosas

...mi profesor trata de comprender como veo las cosas antes de sugerir

6 una nueva forma de hacerlas

$7 \quad$...mi profesor trata de controlar todo lo que hago

$8 \quad$...mi profesor es inflexible

$9 \quad$...mi profesor utiliza un lenguaje fuerte

10 ...mi profesor pone demasiada presión sobre mi

Nota. $\mathrm{AA}=$ Apoyo a la Autonomía; $\mathrm{EC}=$ Estilo Controlador.

La prueba de esfericidad de Barlett fue significativa $\left(\square^{2}=1002.26 \mathrm{gl}=45 ; \mathrm{p}<.001\right) \mathrm{y}$ el estadístico Kaiser-Meyer-Olkin fue mayor a $.50(\mathrm{KMO}=.82)$ lo que indica una adecuación de los datos para realizar un AFE. Los resultados revelaron una estructura factorial formada por 
dos factores (apoyo a la autonomía y estilo controlador) con autovalores superiores a 1 y una varianza total acumulada del $56.12 \%$.

\section{- Análisis Factorial Confirmatorio.}

La Figura 1 representa la estructura de la escala de estilos interpersonales del profesor de educación física. El modelo hipotetiza la existencia de dos variables latentes exógenas denominadas apoyo a la autonomía y estilo controlador. El modelo está compuesto por 10 ítems que se agrupan en dos variables latentes. Los índices de bondad de ajuste del modelo bi-factorial resultó satisfactorio $(\square 2 / \mathrm{gl}=2.90, \mathrm{NNFI}=.95, \mathrm{CFI}=.96, \mathrm{RMSEA}=.08$ y SRMR $=.07)$. El modelo mostró cargas factoriales que oscilan desde .61 hasta .85 para las aspiraciones intrínsecas, en tanto que para las aspiraciones extrínsecas mostraron saturaciones factoriales que oscilan desde .37 hasta .69 (ver Figura 1). Todas las saturaciones factoriales de los ítems resultaron estadísticamente significativas ( $\mathrm{p}<.001$ ), es decir, cada ítem constituye un indicador fiable de la escala de la que forma parte.

\section{- Análisis de consistencia interna.}

El análisis de fiabilidad reveló que la eliminación de ningún ítem mejoraba los coeficientes de fiabilidad, por lo tanto, se mantuvieron todos los ítems de la versión original. Los resultados del análisis de fiabilidad revelaron una consistencia interna buena para el apoyo a la autonomía ( $\square \square=.88$ ), y pobre para el estilo controlador $(\square \square=.55$ ).

Por otro lado, la sub-escala de apoyo a la autonomía presentó una fiabilidad compuesta de .92, superando el valor mínimo aceptable de.70 (Hair et al., 2009), por otra parte, la fiabilidad compuesta de la sub-escala del estilo controlador fue de .82 , por encima de dicho valor criterio. Finalmente, para las dos sub-escalas se logró una varianza media extraída, la sub-escala de apoyo a la autonomía obtuvo un valor de .57 y de .40 para el estilo controlador. 


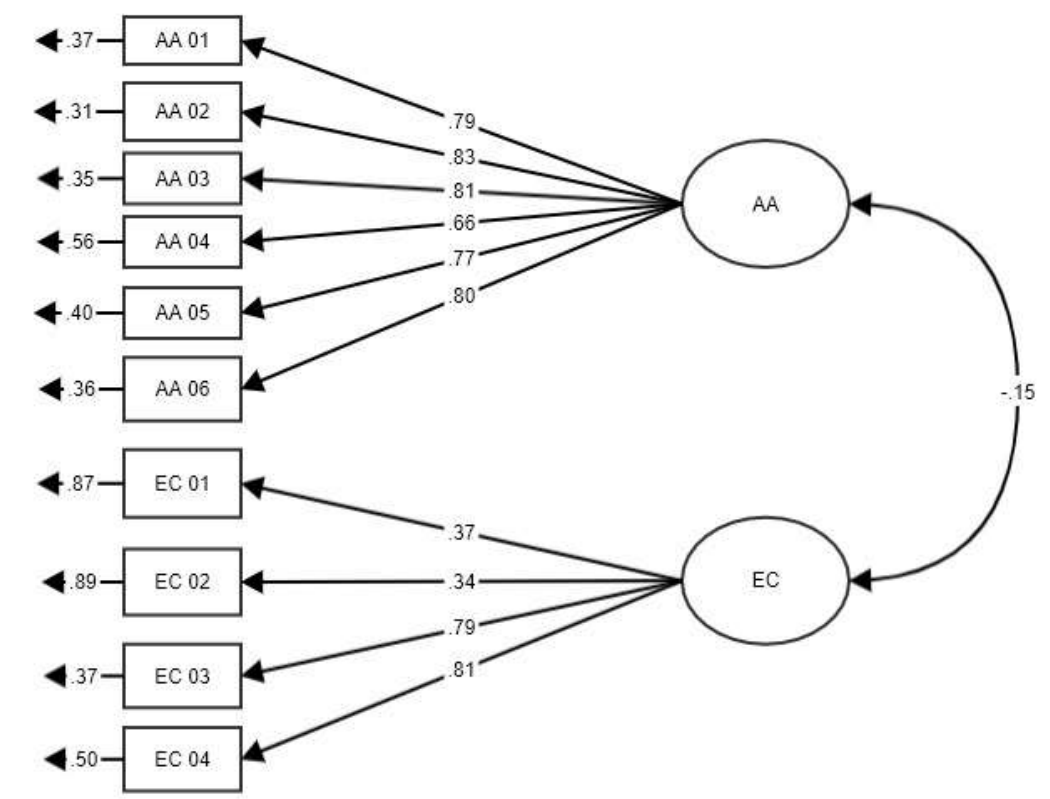

Figura 1. Análisis factorial confirmatorio de la escala de estilos interpersonales del profesor de educación física.

- Motivación. Estadísticos descriptivos.

En la Tabla 6 se muestran los resultados descriptivos de la escala de percepción de locus de causalidad. Los resultados de la asimetría y curtosis revelan una distribución no normal ya que la mayoría de los valores de asimetría y curtosis se encuentran fuera del rango $(-1,1)$.

Tabla 6

Escala de percepción de locus de causalidad. Descriptivos y normalidad

\begin{tabular}{|c|c|c|c|c|c|}
\hline No & Sub escala & $\mathrm{M}$ & DT & Asimetría & Curtosis \\
\hline \multicolumn{6}{|c|}{ Motivación Autónoma } \\
\hline 1 & ...porque es divertida & 5.01 & 2.09 & -.63 & -.88 \\
\hline 2 & ...porque me gusta aprender nuevas habilidades & 4.87 & 2.18 & -.59 & -1.03 \\
\hline 3 & ...porque es emocionante & 4.72 & 2.13 & -.42 & -1.16 \\
\hline 4 & $\begin{array}{l}\text {...porque disfruto como me siento mientras } \\
\text { aprendo nuevas habilidades/técnicas. }\end{array}$ & 4.52 & 2.17 & -.30 & -1.28 \\
\hline 5 & ...porque quiero aprender habilidades deportivas. & 5.04 & 2.06 & -.68 & -.82 \\
\hline 6 & ...porque es importante para mi hacerlo bien & 5.11 & 2.04 & -.72 & -.77 \\
\hline 7 & ...porque quiero mejorar en los deportes & 5.42 & 1.93 & -.97 & -.24 \\
\hline
\end{tabular}


...porque puedo aprender habilidades que tal vez puedan ayudarme en otras áreas de mi vida.

Motivación Controlada

9 ...porque quiero que el (la) maestro (a) piense que soy buen estudiante.

$\begin{array}{llll}4.23 & 2.25 & -.16 & -1.38\end{array}$

10 ...porque me sentiré mal conmigo mismo si no lo

$\begin{array}{llll}2.82 & 2.22 & .83 & -.81\end{array}$

11 ...porque quiero que mis compañeros piensen que

$$
\text { soy hábil }
$$
...porque me incomoda cuando no lo hago.

$\begin{array}{lll}2.71 & 2.17 \quad .92\end{array}$

13 ...porque me meteré en problemas si no lo hago.

$\begin{array}{lll}3.60 & 2.48 \quad .25\end{array}$

No Motivación

$$
\text { ...pero realmente no sé porque lo hago }
$$
...pero no entiendo porque debemos tener esta

$$
\text { clase. }
$$

19 ...pero en realidad siento que pierdo mí tiempo en

$$
\text { esta clase. }
$$
...pero no veo lo que estoy obteniendo de esta

$$
\text { clase. }
$$

Nota. $\mathrm{M}=$ Media, DT = Desviación Típica. Rango: $1-7$. 


\section{- Análisis Factorial Exploratorio (AFE)}

Tabla 7

Escala de percepción de locus de causalidad. Cargas factoriales

Participo en esta clase de educación física...

Cargas

factoriales

No Ítems

MA MC NM

1 ...porque es divertida

.82

2 ...porque me gusta aprender nuevas habilidades

3 ...porque es emocionante

...porque disfruto como me siento mientras aprendo nuevas

4 habilidades/técnicas.

5 ...porque quiero aprender habilidades deportivas.

6 ...porque es importante para mi hacerlo bien

7 ...porque quiero mejorar en los deportes

...porque puedo aprender habilidades que tal vez puedan ayudarme en otras áreas de mi vida.

9 ...porque quiero que el (la) maestro (a) piense que soy buen estudiante.

10 ...porque me sentiré mal conmigo mismo si no lo hago

11 ...porque quiero que mis compañeros piensen que soy hábil

12 ...porque me incomoda cuando no lo hago.

13 ...porque me meteré en problemas si no lo hago.

14 ...porque es lo que se supone que debo hacer.

15 ...para que el (la) maestro(a) no me grite.

16 ...porque es parte del reglamento

17 ...pero realmente no sé porque lo hago

$18 \ldots$...pero no entiendo porque debemos tener esta clase.

19 ...pero en realidad siento que pierdo mí tiempo en esta clase.

20 ...pero no veo lo que estoy obteniendo de esta clase.

Nota. MA = Motivación Autónoma, MC = Motivación Controlada, NM = No Motivación

Siguiendo las recomendaciones de estudios recientes (Lloret-Segura, et al., 2014), para examinar la estructura factorial de los 20 ítems que componen el instrumento para medir los tipos de motivación se realizó un análisis factorial exploratorio utilizando el método de 
factorización de mínimos cuadrados no ponderados (unweighted least squares) y aplicando un criterio de rotación Oblicua (Promax, Kappa =4).

La prueba de esfericidad de Barlett fue significativa $(\square 2=2678.67, \mathrm{gl}=190 ; \mathrm{p}<.001)$ y el estadístico Kaiser-Meyer-Olkin fue mayor a $.50(\mathrm{KMO}=.88)$ lo que indica una adecuación de los datos para realizar un AFE. Los resultados revelaron una estructura factorial formada por tres factores (motivación autónoma, motivación controlada y no motivación) con autovalores superiores a 1 y una varianza total acumulada del $61.78 \%$.

\section{- Análisis Factorial Confirmatorio}

La Figura 2 representa la estructura de la escala de percepción de locus de causalidad. El modelo hipotetiza la existencia de tres variables latentes exógenas denominadas motivación autónoma, motivación controlada y no motivación. El modelo está compuesto por 20 ítems que se agrupan en tres variables latentes. Los índices de bondad de ajuste del modelo tri-factorial resultó satisfactorio $(\square 2 / \mathrm{gl}=2.74, \mathrm{NNFI}=.96, \mathrm{CFI}=.96, \mathrm{RMSEA}=.07$ y SRMR $=.01)$. El modelo mostró cargas factoriales que oscilan desde .69 hasta .84 para la motivación autónoma, desde .56 hasta .71 para la motivación controlada y desde .75 hasta .80 para la no motivación (ver Figura 2). Todas las saturaciones factoriales de los ítems resultaron estadísticamente significativas $(\mathrm{p}<.001)$.

El análisis de fiabilidad reveló que la eliminación de ningún ítem mejoraba los coeficientes de fiabilidad, por lo tanto, se mantuvieron todos los ítems de la versión original. Los resultados del análisis de fiabilidad revelaron una excelente consistencia interna para la motivación autónoma ( $\square=.91$ ), aceptable para la motivación controlada ( $\square=.79$ ) y bueno para la no motivación $(\square=.81)$. 


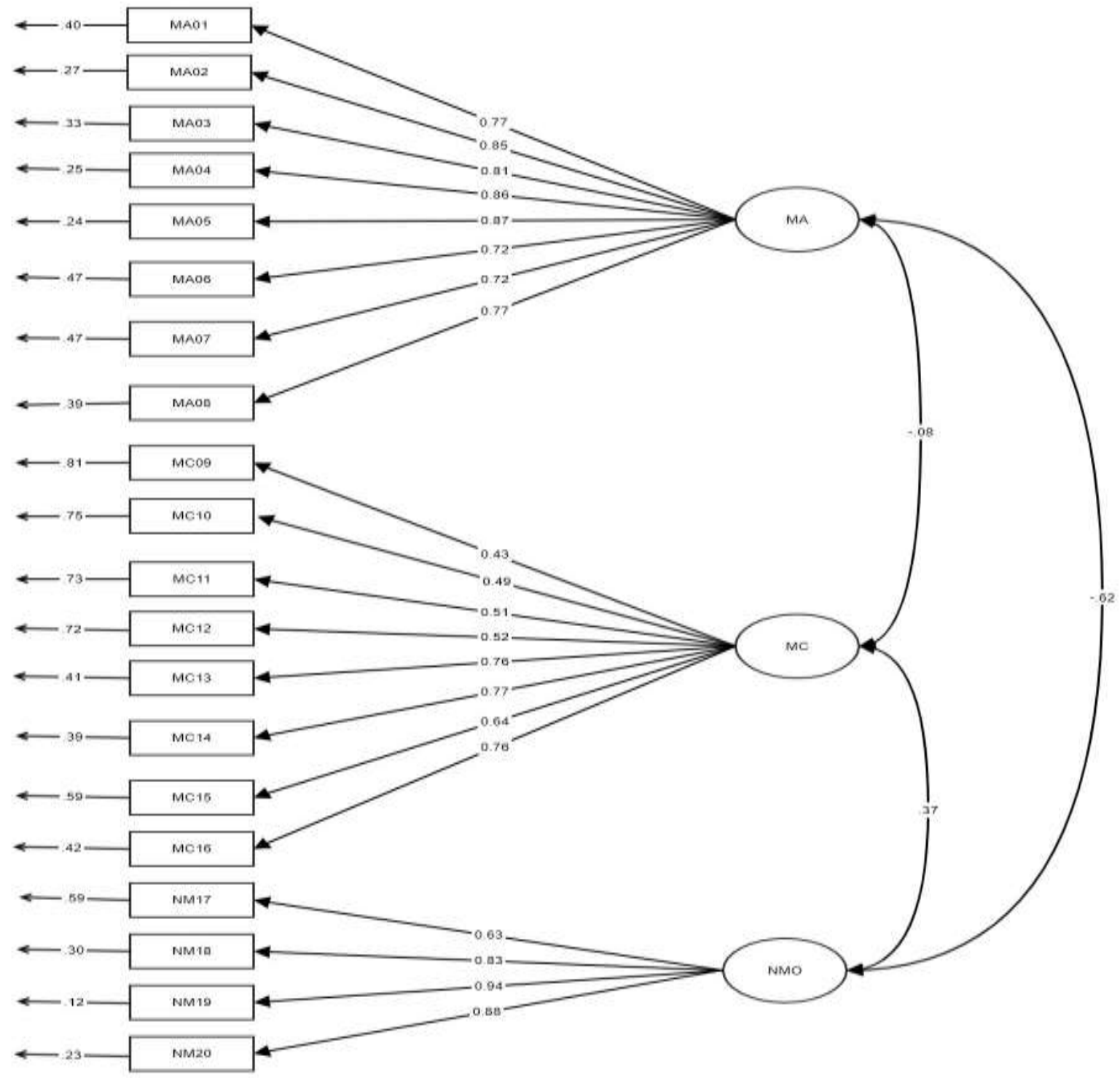

Figura 2. Estructura de la escala de motivación de la clase de educación física.

Por otro lado, la sub-escala de la motivación autónoma presentó una fiabilidad compuesta de .94 , y de .88 para la motivación controlada y no motivación de .91 , es decir, las tres sub-escalas superaron el valor mínimo aceptable de.70 (Hair et al. 2009). Por su parte, las sub-escalas de la motivación autónoma mostro valores adecuados para la varianza media extraída de .60 y motivación controlada .60, no obstante, la sub-escala de la no motivación, obtuvo un coeficiente de .42 , ligeramente por debajo del valor criterio. 
- Estilos interpersonales. Estadísticos descriptivos.

Tabla 8

Ítems que componen la escala de satisfacción/aburrimiento en la educación física

\begin{tabular}{|c|c|c|c|c|c|}
\hline No & Sub escala & $\mathrm{M}$ & DT & Asimetría & Curtosis \\
\hline \multicolumn{6}{|c|}{ Satisfacción/diversión } \\
\hline 1 & $\begin{array}{l}\text { Normalmente me divierto en la clase de } \\
\text { educación física }\end{array}$ & 3.97 & 1.17 & -1.05 & .31 \\
\hline 5 & $\begin{array}{l}\text { Normalmente encuentro la educación física } \\
\text { interesante }\end{array}$ & 3.55 & 1.33 & -.50 & -.90 \\
\hline 6 & $\begin{array}{l}\text { Cuando hago educación física parece que el } \\
\text { tiempo vuela }\end{array}$ & 3.81 & 1.35 & -.91 & -.41 \\
\hline 7 & $\begin{array}{l}\text { Normalmente participo activamente en las } \\
\text { clases de educación física }\end{array}$ & 4.08 & 1.18 & -1.21 & .52 \\
\hline 8 & $\begin{array}{l}\text { Normalmente me lo paso bien haciendo } \\
\text { educación física }\end{array}$ & 4.11 & 1.22 & -1.39 & .99 \\
\hline \multicolumn{6}{|c|}{ Aburrimiento } \\
\hline 2 & $\begin{array}{l}\text { En las clases de educación física a menudo } \\
\text { sueño despierto en vez de pensar en lo que } \\
\text { hago realmente }\end{array}$ & 2.24 & 1.29 & .65 & -.71 \\
\hline 3 & $\begin{array}{l}\text { En las clases de educación física normalmente } \\
\text { me aburro }\end{array}$ & 2.47 & 1.43 & .45 & -1.16 \\
\hline 4 & $\begin{array}{l}\text { En educación física deseo que la clase termine } \\
\text { rápidamente }\end{array}$ & 1.97 & 1.35 & 1.13 & -.08 \\
\hline
\end{tabular}

Nota. $\mathrm{M}=$ Media, DT = Desviación Típica. Rango: $1-5$. 
En la Tabla 8 se muestran los resultados descriptivos de la escala satisfacción/aburrimiento en la clase de educación física. Los resultados de la asimetría y curtosis revelan una distribución no normal ya que la mayoría de los valores de asimetría y curtosis se encuentran fuera del rango $(-1,1)$.

- Análisis Factorial Exploratorio (AFE)

Siguiendo las recomendaciones de estudios recientes (Lloret-Segura et al. 2014), para examinar la estructura factorial de los ocho ítems que componen el instrumento para medir la satisfacción/diversión y aburrimiento, se realizó un análisis factorial exploratorio utilizando el método de factorización de mínimos cuadrados no ponderados (unweighted least squares) y aplicando un criterio de rotación Oblicua (concretamente Promax, Kappa =4).

La prueba de esfericidad de Barlett fue significativa $(\square 2=861.47, \mathrm{gl}=28$; $\mathrm{p}<.001) \mathrm{y}$ el estadístico Kaiser-Meyer-Olkin fue mayor a $.50(\mathrm{KMO}=.88)$ lo que indica una adecuación de los datos para realizar un AFE. Los resultados revelaron una estructura factorial formada por dos factores (Satisfacción/Diversión y Aburrimiento) con autovalores superiores a 1 y una varianza total acumulada del 50.19\%. 
Tabla 9

Escala de satisfacción en la educación física. Cargas factoriales

En la clase de educación física ...

Cargas

factoriales

No Ítem

$\mathrm{S} / \mathrm{D} \quad \mathrm{A}$

1 Normalmente me divierto en la clase de educación física

.85

5 Normalmente encuentro la educación física interesante

6 Cuando hago educación física parece que el tiempo vuela

Normalmente participo activamente en las clases de educación física

En las clases de educación física a menudo sueño despierto en vez de pensar en lo que hago realmente

3 En las clases de educación física normalmente me aburro

4 En educación física deseo que la clase termine rápidamente

Nota. S/D = Satisfacción/Diversión; A = Aburrimiento.

\section{- Análisis Factorial Confirmatorio}

La Figura 3 representa la estructura de la escala de satisfacción intrínseca de la clase de educación física. El modelo hipotetiza la existencia de dos variables latentes denominadas satisfacción/diversión y aburrimiento. El modelo está compuesto por ocho ítems que se agrupan en dos variables latentes. Los índices de bondad de ajuste del modelo bi-factorial resultó satisfactorio $(\square 2 / \mathrm{gl}=1.37, \mathrm{NNFI}=.99, \mathrm{CFI}=.99, \mathrm{RMSEA}=.03$ y $\mathrm{SRMR}=.03) . \mathrm{El}$ modelo mostró cargas factoriales que oscilan desde .29 hasta .69 para la satisfacción/diversión y desde .42 hasta .89 para el aburrimiento (ver Figura 3). Todas las saturaciones factoriales de los ítems resultaron estadísticamente significativas $(\mathrm{p}<.001)$. 


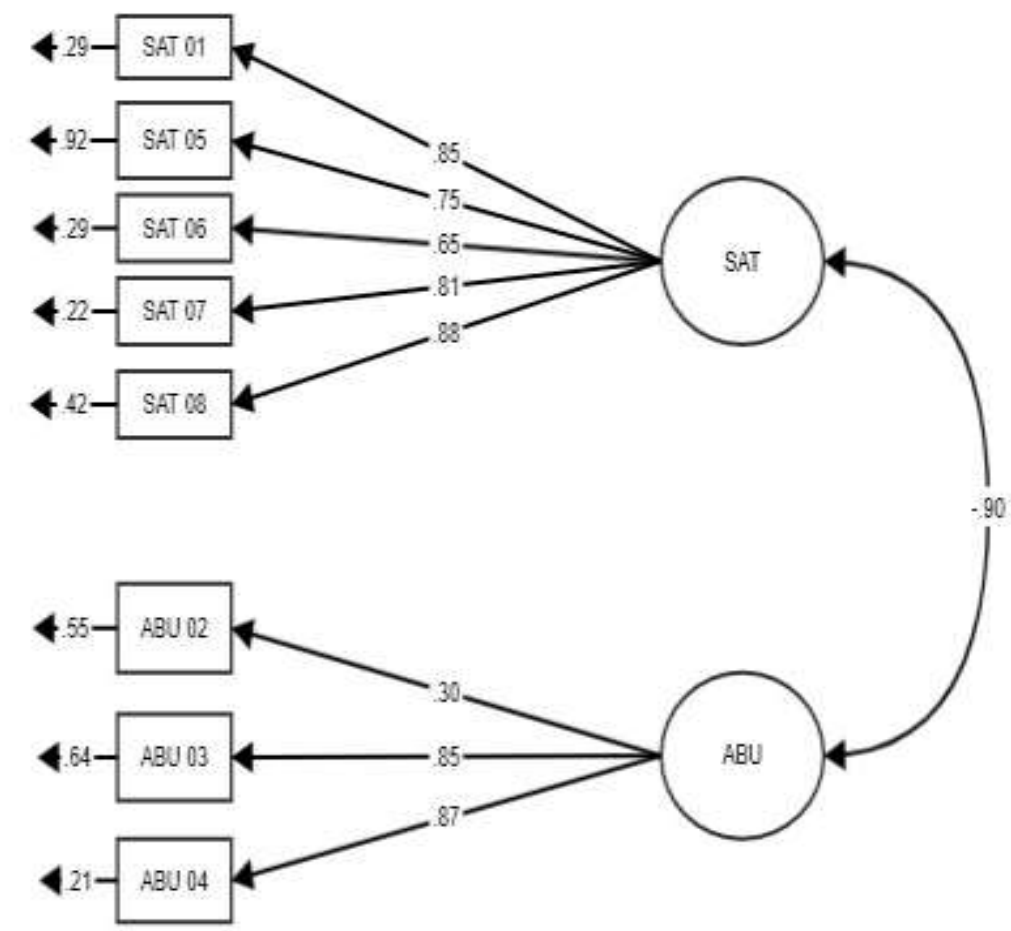

Figura 3. Estructura de la escala de satisfacción intrínseca de la clase de educación física.

\section{- Análisis de consistencia interna}

El análisis de fiabilidad reveló que la eliminación de ningún ítem mejoraba los coeficientes de fiabilidad, por lo tanto, se mantuvieron todos los ítems de la versión original. Los resultados del análisis de fiabilidad revelaron una consistencia interna entre buena y cuestionable para la satisfacción/diversión ( $\square=.82$ ), y el aburrimiento ( $\square=.64$ ).

No obstante, tanto la sub-escala de satisfacción/diversión como la de aburrimiento presentaron una fiabilidad compuesta por encima del valor mínimo aceptable (Hair, et al., 2009) de .90 para la satisfacción/diversión y de .85 para el aburrimiento. Finamente, el valor de la varianza media extraída de la sub-escala de aburrimiento fue de .54 , es decir, por encima del valor mínimo aceptable, lo mismo sucedió con la sub-escala de satisfacción/diversión con un valor $(\mathrm{AVE}=.51)$. 


\section{- Correlaciones entre variables}

Tabla 10

Descriptivos y correlaciones entre las variables del estudio.

\begin{tabular}{lllllllll}
\hline & $\mathrm{M}$ & $\mathrm{DT}$ & 1 & 2 & 3 & 4 & 5 & 6 \\
\hline 1. Apoyo a la Autonomía & 3.67 & 1.67 & 1 & & & & & \\
2. Estilo Controlador & 3.07 & 1.33 & -.07 & 1 & & & & \\
3. Motivación Autónoma & 4.95 & 1.64 & $.52^{* *}$ & -.07 & 1 & & & \\
4. Motivación Controlada & 3.47 & 1.44 & -.01 & $.17^{* *}$ & .03 & 1 & & \\
5. No Motivación & 2.27 & 1.58 & $-.34^{* *}$ & $.17^{* *}$ & $.26^{* *}$ & $.26^{* *}$ & 1 & \\
6. Satisfacción/Diversión & 3.90 & .95 & $.47^{* *}$ & -.04 & $.74^{* *}$ & -.04 & $-.45^{* *}$ & 1 \\
7. Aburrimiento & 2.23 & 1.03 & $-.35^{* *}$ & $.21^{* *}$ & $-.55^{* *}$ & $.12^{*}$ & $.47^{* *}$ & $-.64^{* *}$
\end{tabular}

Nota. ${ }^{*} *$ La correlación es significativa al nivel 0,01 (bilateral)

* La correlación es significativa al nivel 0,05 (bilateral)

Previo a la prueba del modelo hipotetizado, se realizó un análisis de correlación entre las variables involucradas en el estudio. Los resultados se muestran en la Tabla 10 donde se destaca las relaciones positivas entre el apoyo a la autonomía, la motivación autónoma y la satisfacción/diversión, las relaciones positivas entre el estilo controlador, la motivación controlada, la no motivación y el aburrimiento. La relación positiva entre la motivación autónoma y la satisfacción/diversión, y negativa con el aburrimiento, así como la relación positiva entre la no motivación y el aburrimiento.

\section{Discusión y conclusiones}

Finalmente, los resultados de este estudio permiten generalizar los postulados de la teoría de la autodeterminación a otras poblaciones y contextos culturales, como es el mexicano. Cuando los estudiantes de secundaria reciben sus clases de EF de forma que el maestro promueve la autonomía, hay un mayor disfrute de la clase. 
Los profesores de EF, deben considerar con gran atención el diseño y operación de actividades que permitan o fomenten entre los estudiantes el apoyo a la autonomía, ya que será un contexto social, que impacte positivamente en la motivación intrínseca, y ésta a su vez, será un excelente mediador para generar resultantes positivas, como la Satisfacción/Diversión, se debe de analizar de la misma manera eliminar o excluir actividades que solo generan malestar, en las cuales el alumno no encuentra una motivación para efectuarlas y esto a su vez ocasiona el Aburrimiento.

Este estudio presenta algunas limitaciones relativas a las características específicas de la muestra de estudio ya que se trata de estudiantes de secundarias en el municipio de Hermosillo. No obstante, estas limitaciones indican posibles direcciones para futuras investigaciones, ya que sería interesante ampliar el estudio con alumnos de primaria y realizar una recogida longitudinal de datos para fortalecer las conclusiones sobre las relaciones de predicción de las variables incluidas en el estudio.

\section{Agradecimientos}

Se agradece al Programa para el Desarrollo Profesional Docente, para el tipo superior (PRODEP) por financiar este proyecto de investigación.

\section{Referencias.}

Assor, A., Kaplan, H., \& Roth, G. (2002). Choice is good, but relevance is excellent: Autonomy-enhancing and suppressing teaching behaviors predicting students' engagement in schoolwork. British Journal of Educational Psychology, 27, 261-278.

Baena-Extremera, A., \& Granero-Gallegos, A., \& Bracho- Amador, C., \& Pérez-Quero, F. (2012). Spanish Version of the Sport Satisfaction Instrument (SSI) Adapted to Physical Education. Revista de Psicodidáctica, 17 (2), 377-396.

Balaguer, I., Atienza, F. L., Castillo, I., Moreno, Y., \& Duda, J. L. (1997). Factorial structure of measures of satisfaction/interest in sport and classroom in the case of Spanish adolescents. En 4th European Conference of Psychological Assessment (p. 76). Lisbon: Portugal.

Bartholomew, K., Ntoumanis, N., y Thøgersen-Ntoumani, C. (2011). Self-Determination theory and the darker side of athletic experience: The role of interpersonal control and need thwarting. Sport and Exercise Psychology Review,7, 23-27.

Brasó Rius, J., \& Torrebadella Flix, X. (2018). El juego del marro. Un dispositivo de la pedagogía disciplinar en la tradición inventada de la educación física contemporánea del siglo XIX y principios del XX. Lúdica Pedagógica, 1(27). https://doi.org/10.17227/ludica.num27-9443. 
Brislin, R. W. (1986). The wording and translation of research instruments.

Cheon, S. H., \& Reeve, J. (2015). A classroom-based intervention to help teachers decrease students' amotivation. Contemporary educational psychology, 40, 99-111.

Cheon, S. H., Reeve, J., Yu, T. H., \& Jang, H. R. (2014). Teacher benefi ts from giving students autonomy support during physical education instruction. Journal of Sport and Exercise Psychology, 36, 331-346.

Deci, E. L., \& Ryan, R. M. (1985). Intrinsic motivation and self-determination in human behavior. New York: Plenum.

Deci, E. L., \& Ryan, R. M. (1985). The general causality orientations scale: Selfdetermination in personality. Journal of research in personality, 19(2), 109-134.

Deci, E. L., \& Ryan, R. M. (2000). The" what" and" why" of goal pursuits: Human needs and the self-determination of behavior. Psychological inquiry, 11(4), 227-268.

Deci, E. L., \& Ryan, R. M. (2002). Overview of self-determination theory: An organismic dialectical perspective. Handbook of self-determination research, 3-33.

Deci, E. L., \& Ryan, R. M. (2008). Facilitating optimal motivation and psychological wellbeing across life's domains. Canadian Psychology/Psychologie canadienne, 49(1), 1423.

Goudas, M., Biddle, S., \& Fox, K. (1994). Perceived locus of causality, goal orientations, and perceived competence in school physical-education classes. British Journal of Educational Psychology, 64(3), 453-463.

Gunnell, K. E., Crocker, P. R., Wilson, P. M., Mack, D. E., \& Zumbo, B. D. (2013). Psychological need satisfaction and thwarting: A test of basic psychological needs theory in physical activity contexts. Psychology of Sport and Exercise, 14(5), 599-607.

Hair, J. F., Black, W. C., Babin, B. J., Anderson, R. E., \& Tatham, R. L. (2009). Análise multivariada de dados. Bookman editora.

Jang, H., Kim, E. J., \& Reeve, J. (2016). Why students become more engaged or more disengaged during the semester: A self-determination theory dual-process model. Learning and Instruction, 43, 27-38.

Jang, H., Reeve, J., \& Deci, E. L. (2010). Engaging students in learning activities: It is not autonomy support or structure but autonomy support and structure. Journal of Educational Psychology, 102(3), 588-600. https://doi.org/10.1037/a0019682.

Jung, C. G. (2013). The psychology of the transference. Routledge.

Lloret-Segura, S., Ferreres-Traver, A., Hernández-Baeza, A., \& Tomás-Marco, I. (2014). El análisis factorial exploratorio de los ítems: una guía práctica, revisada y actualizada. Anales de Psicología/Annals of Psychology, 30(3), 1151-1169. 
Maldonado Maldonado, E., Pacheco Ríos, R., \& Zamarripa, J. (2017). Validación mexicana del cuestionario de clima de aprendizaje adaptado a la educación física (Mexican validation of learning climate questionnaire for physical education). Retos, 32, 115 118. https://recyt.fecyt.es/index.php/retos/article/view/55170.

Márquez, J. M. C., \& Celis, C. C. (2016b). El crecimiento y el desarrollo neuromotor, óseo y muscular en la edad escolar. Wanceulen S.L.

Martela, F., DeHaan, C. R., \& Ryan, R. M. (2016). On enhancing and diminishing energy through psychological means: Research on vitality and depletion from selfdetermination theory. En E. Hirt (Ed.), Self-regulation and ego control. New York: Elsevier.

Miller, J. G., Das, R., \& Chakravarthy, S. (2011). Culture and the role of choice in agency. Journal of Personality and Social Psychology, 101, 46-61.

Muñiz, J. (2000). Teoría Clásica de los Tests. Madrid: Pirámide

Muñiz, J., \& Bartram, D. (2007). Improving international tests and testing. European Psychologist, 12(3), 206-219.

Osterlind, S.J. (1989). Constructing tests items. Boston: Kluwer Academic Publishers.

Reeve, J. (2009). Why teachers adopt a controlling motivating style toward students and how they can become more autonomy supportive. Educational Psychologist, 44 (3), 159175. doi: 10.1080/00461520903028990.

Reeve, J. (2016). Autonomy-supportive teaching: What it is, how to do it. In Building autonomous learners (pp. 129-152). Springer, Singapore.

Reeve, J., \& Jang, H. S. (2006). What teachers say and do to support students' autonomy during a learning activity. Journal of Educational Psychology, 98 (1), 209-218. doi: 10.1037/0022-0663.98.1.209.

Ryan, R. M. (1982). Control and information in the intrapersonal sphere: An extension of cognitive evaluation theory. Journal of Personality and Social Psychology, 43 (3), 450-461. doi: 10.1037//0022-3514.43.3.450.

Soenens, B., \& Vansteenkiste, M. (2010). A theoretical upgrade of the concept of parental psychological control: Proposing new insights on the basis of self-determination theory. Developmental Review, 30 (1), 74-99. doi: 10.1016/j.dr.2009.11.001.

Tapia López, A. (2019). Diferencias en los niveles de actividad física, grado de adherencia a la dieta mediterránea y autoconcepto físico en adolescentes en función del sexo (Gender differences in physical activity levels, degree of adherence to the Mediterranean diet, and physi. Retos: Nuevas tendencias en Educación Física, Deporte y Recreación, 36(36), 185-192. https://recyt.fecyt.es/index.php/retos/article/view/67130. 
Vansteenkiste, M., Simons, J., Lens, W., Soenens, B., \& Matos, L. (2005). Examining the motivational impact of intrinsic versus extrinsic goal framing and autonomysupportive versus internally controlling communication style on early adolescents' academic achievement. Child Development, 76, 483-501. doi: 10.1111/j.14678624.2005.00858.x.

Vázquez, P. G., \& Márquez, A. R. (2017). La actividad física como fuente de salud y calidad de vida. Sevilla: Wanceulen.

Williams, G. C., \& Deci, E. L. (1996). Internalization of biopsychosocial values by medical students: A test of self-determination theory. Journal of Personality and Social Psychology, 70(4), 767-779.

Zamarripa, J., Castillo, I., Tomás, I., Tristán, J., \& Álvarez, O. (2016). El papel del profesor en la motivación y la salud mental de los estudiantes de educación física. Salud mental, 39(4), 221-227.

Zougkou, K., Weinstein, N., \& Paulmann, S. (2017). ERP correlates of motivating voices: Quality of motivation and time-course matters. Social Cognitive and Affective Neuroscience, 12(10), 1687-1700.

Zueck, M. C., Ramírez, A. A., Rodríguez, J. M., \& Irigoyen, H. E. (2020). Satisfacción en las clases de Educación Física y la intencionalidad de ser activo en niños del nivel de primaria. Retos: Nuevas tendencias en Educación Física, Deporte y Recreación, 37, 33-40. https://recyt.fecyt.es/index.php/retos/article/view/69027.

Cómo citar:

Duarte Félix, H., Castro Zamora, A. ., Borbón Castro, N. ., De La Cruz-Ortega , M. ., \& Durazo Terán, L. (2021). Relación de los estilos interpersonales del profesor y la motivación, diversión y aburrimiento del estudiante en la clase de educación física en escuelas secundarias de Hermosillo, Sonora, México. Revista De Investigación Académica Sin Frontera: División De Ciencias Económicas Y Sociales, (36).

https://doi.org/10.46589/rdiasf.vi36.410

Ciencias Económicas Y Sociales, (36). https://doi.org/10.46589/rdiasf.vi36.425

\section{CRIS - UNISON}

Sistema de Gestión de la Investigación

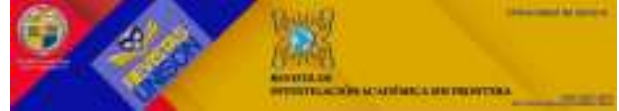

D Dialnet lationde解

IfF IIIFACTOR 